\title{
Dietary questions as determinants of mortality: the OXCHECK experience
}

\author{
David Whiteman ${ }^{1, *}$, John Muir ${ }^{1}$, Lesley Jones ${ }^{1}$, Mike Murphy ${ }^{1}$ and Tim Key ${ }^{2}$ \\ 'ICRF General Practice Research Group, Institute of Health Sciences, Old Road, Oxford OX3 7LF, UK: \\ ${ }^{2}$ ICRF Cancer Epidemiology Unit, Radcliffe Infirmary, Oxford OX2 6HE, UK
}

Submitted 26 November 1998: Accepted 19 March 1999

\begin{abstract}
Objective: To determine whether responses to simple dietary questions are associated with specific causes of death.

Design: Self-reported frequency intakes of various classes of foods and data on confounding factors were collected at the baseline survey. Death notifications up to 31 December 1997 were ascertained from the Office for National Statistics. Relative risk (RR) of death and 95\% confidence intervals (CI) associated with baseline dietary factors were calculated by Cox regression.

Setting: Prospective follow-up study based on five UK general practices.

Subjects: Data were used from 11090 men and women aged 35-64 years (81\% of the eligible patient population) who responded to a postal questionnaire in 1989.

Results: After 9 years of follow-up, 598 deaths were recorded, 514 of these among the 10522 subjects with no previous history of angina. All-cause mortality was positively associated with age, smoking and low social class, as expected. Among the dietary variables, all-cause mortality was significantly reduced in participants who reported relatively high consumption of vegetables, puddings, cakes, biscuits and sweets, fresh or frozen red meat (but not processed meat), among those who reported using polyunsaturated spreads and among moderate alcohol drinkers. These associations were broadly similar for deaths from ischaemic heart disease (IHD), cancer and all other causes combined, and were not greatly attenuated by adjusting for potential confounding factors including social class.

Conclusions: Responses to simple questions about nutrition were associated with mortality. These findings must be interpreted with caution since residual confounding by dietary and lifestyle factors may underlie the associations.
\end{abstract}

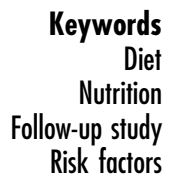

The complex relationship between diet and health is of enduring importance to consumers, health practitioners and policy makers. While the role of specific nutrients in the aetiology (and prevention) of common diseases is currently the focus of intense research ${ }^{1}$, there remains a gulf between defining these complex and interrelated biochemical pathways, and the practicalities of population-wide dietary modification. Few reports have been published relating disease incidence or mortality among industrialized populations to consumption of broad food groups (e.g. vegetables, red meat, milk, fruit), yet most nonnutritionists are likely to perceive their dietary intake in this way.

To address the question of whether simple dietary assessments are associated with risk of death from specific causes, we analysed the mortality experience of a prospective study of more than 11000 patients in a British primary care setting with 9 years' follow-up.

\section{Methods}

\section{Subjects}

The study population consisted of all those who participated in the baseline data collection for the OXCHECK study, a randomized trial of nurse health checks in primary care. A detailed description of the protocol has been provided in an earlier report ${ }^{2}$. Briefly, in 1989 questionnaires were posted to all 17965 patients registered with five urban practices around Luton and Dunstable (Bedfordshire, UK) who were aged 35-64 years on 1 January 1989. Of these, 338 were ineligible to participate and 3810 were subsequently found to have died or no longer lived at the address recorded by their registered practice. After three mailings, 11090 patients (80.3\% of available subjects) returned a completed questionnaire, 531 refused to participate (3.8\%) and 2196 (15.9\%) did not respond to the baseline survey ${ }^{2}$. The completed 
responses form the basis of this report. Of the 11090 respondents to the initial mailing, 8109 subsequently participated in the OXCHECK trial.

\section{Data collection}

Data were collected by self-completed questionnaires mailed to all participants prior to participation in the OXCHECK trial. As well as background information regarding occupation, education and smoking, respondents completed a series of validated questions to identify those with angina or a past history of possible myocardial infarction ${ }^{3}$. In addition, subjects were asked 'Do you have any long-standing illness, disability or infirmity (that has troubled you over a period of time or is likely to affect you over a period of time)?', and if so, to specify the diagnosis. Dietary details were elicited by a series of simple food frequency questions modified from a 'health and lifestyle survey' ${ }^{4}$. Participants were given a list of foods (Appendix 1) and asked 'About how often do you eat the following foods'; permissible responses were 'never', 'less than once a week', '1-3 days a week' or '4-7 days a week'. A measure of weekly alcohol consumption was derived by multiplying the average weekly frequency of drinking beer, wine, fortified wine and spirits by the typical quantity consumed on such occasions. The respondent's height, weight and frequency of participation in vigorous exercise were elicited. The body mass index (BMI) was derived for each participant as weight in kilograms divided by the square of their height in metres. Social class was assigned according to the
Occupational Classifications of the Office of Population Censuses and Surveys 5 .

\section{Follow-up}

All respondents to the 1989 survey were 'flagged' with the Office for National Statistics National Health Service Central Register (ONS NHSCR) for continuous follow-up for mortality and cancer incidence. Death certificates were obtained and cause of death determined according to ONS ICD-9 coding practice. For the analyses in this paper, deaths were assigned to three mutually exclusive categories, namely IHD (ICD-9 codes 410.0-414.9), all cancers (ICD-9 codes 140.0239.9) and other causes of death (all other ICD-9 categories).

\section{Analysis}

To describe the overall mortality of the cohort, standardized mortality ratios for deaths from all causes were calculated using national mortality data for England and Wales. The relative risk (and 95\% confidence intervals) of dying associated with each level of exposure to dietary factors was estimated by the hazard ratio in a series of Cox regression models. Of the food groups assessed in the dietary questionnaire, the following were hypothesized a priori to be associated with a higher risk of mortality at higher levels of consumption: processed meats; fresh or frozen red meat; chips; and biscuits, cakes, puddings or sweets (collectively). Conversely, frequent consumption of fresh or frozen green vegetables, fresh fruit or fruit

Table 1a Causes of death among 278 male participants (with no previous history of angina) who died during follow-up, according to category

\begin{tabular}{|c|c|c|c|c|c|c|c|}
\hline \multicolumn{2}{|c|}{ All causes $(n=278)$} & \multicolumn{2}{|c|}{$\begin{array}{l}\text { Ischaemic heart disease } \\
\qquad(n=71)\end{array}$} & \multicolumn{2}{|c|}{ All cancers $(n=114)$} & \multicolumn{2}{|l|}{ Others $(n=93)$} \\
\hline Disease & No. (\%) & Disease & No. (\%) & Disease & No. (\%) & Disease & No. (\%) \\
\hline Acute MI & 47 (16.9) & Acute MI & 47 (66.2) & Lung & $22(19.3)$ & Stroke & $15(16.1)$ \\
\hline Chronic IHD & $24(8.6)$ & Chronic IHD & $24(37.8)$ & Unknown primary & $17(14.9)$ & COAD & $12(12.9)$ \\
\hline Lung ca. & $22(7.9)$ & & & Prostate & $11(9.6)$ & Chronic liver disease & $7(7.5)$ \\
\hline Ca. unknown site & $17(6.1)$ & & & Colon & $10(8.8)$ & Diabetes & $4(4.3)$ \\
\hline Stroke & $15(5.4)$ & & & Stomach & $8(7.0)$ & Pneumonia & $4(4.3)$ \\
\hline COAD & $12(4.3)$ & & & Pancreas & $7(6.1)$ & Cardiomyopathy/valvular disease & $4(4.3)$ \\
\hline
\end{tabular}

Table 1b Causes of death among 236 female participants (with no previous history of angina) who died during follow-up, according to category

\begin{tabular}{|c|c|c|c|c|c|c|c|}
\hline \multicolumn{2}{|c|}{ All causes $(n=236)$} & \multicolumn{2}{|c|}{$\begin{array}{l}\text { Ischaemic heart disease } \\
\qquad(n=36)\end{array}$} & \multicolumn{2}{|c|}{ All cancers $(n=121)$} & \multicolumn{2}{|c|}{ Others $(n=79)$} \\
\hline Disease & No. (\%) & Disease & No. (\%) & Disease & No. (\%) & Disease & No. (\%) \\
\hline Breast ca. & $34(14.4)$ & Acute MI & $28(77.8)$ & Breast & $34(28.1)$ & Stroke & $20(25.3)$ \\
\hline Acute MI & $28(11.9)$ & Chronic IHD & $8(22.2)$ & Lung & $18(14.9)$ & Pneumonia & $5(6.3)$ \\
\hline Stroke & $20(8.5)$ & & & Unknown primary & $12(10.0)$ & Heart valves & $5(6.3)$ \\
\hline Lung ca. & $18(7.6)$ & & & Stomach & $11(9.1)$ & Asthma & $4(5.1)$ \\
\hline Ca. unknown site & $12(5.1)$ & & & Ovary & $10(8.3)$ & Chronic lung & $3(3.8)$ \\
\hline Stomach ca. & $11(4.7)$ & & & Colon & $7(5.7)$ & Diabetes & $3(3.8)$ \\
\hline
\end{tabular}

ca., carcinoma; COAD, chronic obstructive airways disease; IHD, ischaemic heart disease; MI, myocardial infarction. Stroke refers to deaths coded as any of intracerebral haemorrhage, cerebral infarction, cerebrovascular accident or subarachnoid haemorrhage. 
juice, skimmed milk, polyunsaturated margarine and fresh or frozen fish were each hypothesized to be associated with reduced mortality risks. Relative risks were separately estimated for all-cause mortality and for the three specific categories of death (cause-specific mortality). The person-time contribution of each subject was calculated from the date of return of the questionnaire until the date of death, or until 31 December 1997 for surviving subjects. For 56 subjects $(0.5 \%)$, observations were censored at the date of emigration from the UK.

Dietary exposures were analysed as categorical variables, with data for intake frequency groups 'never' and 'less than once a week' collapsed to form the reference category. For alcohol consumption, abstainers formed the reference category, light drinkers were defined as males who drank no more than 20 units per week or females who drank no more than 15 units per week; participants with higher levels of consumption were classified as moderate to heavy drinkers. Because patients with a prior history of angina or myocardial infarction may have modified their diet just prior to baseline dietary collection, dietary analyses were restricted to the 10522 patients who reported no previous episodes of chest pain. Confounding and effect modification were investigated by conducting analyses stratified by the exposure levels of other risk factors. Where no effect modification was observed across strata, possible confounding was controlled through the inclusion of design variables in multivariable Cox regression analyses. All analyses were conducted in PROC PHREG of SAS v6.12 ${ }^{6}$.

\section{Results}

\section{Mortality}

As of 31 December 1997, 598 participants had died of the following causes: IHD 144 (24.1\%), all cancers 257 (43.0\%) and other causes 197 (32.9\%). Standardized mortality ratios for all-cause mortality were 0.75 for men, 0.87 for women and 0.80 for the entire cohort. Among the 10522 subjects with no prior history of angina there were 514 deaths attributed to: IHD 107 (20.8\%), all cancers 235 (45.7\%) and other causes 172

Table 2 Distribution of established risk factors at baseline among patients with no previous history of angina $(n=10522)$, and crude relative risks $(95 \% \mathrm{Cl})$ of death from all causes and from specific causes, associated with levels of exposure

\begin{tabular}{|c|c|c|c|c|c|}
\hline Variable & No. $(\%)^{*}$ & All-cause mortality & IHD mortality & Cancer mortality & Other mortality \\
\hline \multicolumn{6}{|l|}{ Gender } \\
\hline Male & $4929(46.8)$ & 1.00 & 1.00 & 1.00 & 1.00 \\
\hline Female & $5593(53.2)$ & $0.74(0.62-0.88)$ & $0.44(0.30-0.66)$ & $0.93(0.82-1.20)$ & $0.74(0.54-0.99)$ \\
\hline \multicolumn{6}{|l|}{ Age group (years) } \\
\hline $35-44$ & 4035 (38.3) & 1.00 & 1.00 & 1.00 & 1.00 \\
\hline $45-54$ & $3418(32.5)$ & $3.76(2.67-5.30)$ & $3.59(1.31-9.89)$ & $5.05(3.02-8.45)$ & $2.63(1.55-4.47)$ \\
\hline $55-64$ & $3069(29.2)$ & $10.78(7.85-14.81)$ & $24.14(9.80-59.46)$ & $10.83(6.63-17.68)$ & $7.41(4.59-11.94)$ \\
\hline \multicolumn{6}{|l|}{ Smoking } \\
\hline Never & 4089 (38.9) & 1.00 & 1.00 & 1.00 & 1.00 \\
\hline Ex-smoker & $3175(30.2)$ & $1.71(1.36-2.16)$ & $3.41(1.80-6.49)$ & $1.77(1.28-2.45)$ & $1.20(0.81-1.79)$ \\
\hline Pipe/cigar & $533(5.1)$ & $1.94(1.32-2.86)$ & $3.58(1.36-9.43)$ & $1.33(0.70-2.53)$ & $2.28(1.28-4.06)$ \\
\hline Cigarettes $<15$ day $^{-1}$ & $839(8.0)$ & $2.44(1.80-3.30)$ & $9.30(4.73-18.26)$ & $1.65(1.01-2.70)$ & $1.68(0.97-2.90)$ \\
\hline Cigarettes $15-24$ day $^{-1}$ & $1335(12.7)$ & $2.12(1.61-2.78)$ & $5.04(2.52-10.07)$ & $1.90(1.28-2.83)$ & $1.65(1.04-2.63)$ \\
\hline Cigarettes $25+$ day $^{-1}$ & $539(5.1)$ & $2.04(1.40-2.98)$ & $5.32(2.27-12.44)$ & $1.44(0.78-2.67)$ & $1.96(1.06-3.60)$ \\
\hline \multicolumn{6}{|l|}{ Long-standing disease } \\
\hline No & $6992(67.6)$ & 1.00 & 1.00 & 1.00 & 1.00 \\
\hline Yes & $3355(32.4)$ & $2.83(2.37-3.37)$ & $4.12(2.74-6.20)$ & $1.97(1.52-2.55)$ & $3.75(2.74-5.12)$ \\
\hline \multicolumn{6}{|l|}{ Participation in OXCHECK } \\
\hline No & $2744(26.3)$ & 1.00 & 1.00 & 1.00 & 1.00 \\
\hline Yes & $7704(73.7)$ & $0.60(0.49-0.72)$ & $0.51(0.34-0.77)$ & $0.70(0.52-0.94)$ & $0.52(0.38-0.73)$ \\
\hline \multicolumn{6}{|l|}{ Social class } \\
\hline$|-| \mid$ & $2539(26.0)$ & 1.00 & 1.00 & 1.00 & 1.00 \\
\hline III & $4464(45.7)$ & $1.46(1.12-1.90)$ & $2.15(1.14-4.05)$ & $1.37(0.94-2.00)$ & $1.26(0.78-2.02)$ \\
\hline IV-V & $1762(18.1)$ & $2.15(1.60-2.88)$ & $3.54(1.81-6.93)$ & $1.58(1.02-2.45)$ & $2.34(1.42-3.86)$ \\
\hline Other & $996(10.2)$ & $2.91(2.13-3.98)$ & $2.85(1.30-6.25)$ & $2.35(1.48-3.73)$ & $3.79(2.27-6.31)$ \\
\hline \multicolumn{6}{|l|}{$\mathrm{BMI}\left(\mathrm{kg} \mathrm{m}^{-2}\right)$} \\
\hline$<25$ & $5973(57.8)$ & 1.00 & 1.00 & 1.00 & 1.00 \\
\hline $25-29$ & $3453(33.4)$ & $1.03(0.85-1.24)$ & $1.73(1.14-2.61)$ & $0.92(0.69-1.21)$ & $0.86(0.61-1.22)$ \\
\hline $30+$ & $902(8.7)$ & $1.01(0.73-1.39)$ & $1.61(0.83-3.12)$ & $0.60(0.34-1.06)$ & $1.33(0.81-2.18)$ \\
\hline \multicolumn{6}{|l|}{ Vigorous exercise } \\
\hline Never $/<$ once month $^{-1}$ & $7347(71.5)$ & 1.00 & 1.00 & 1.00 & 1.00 \\
\hline$<$ Once week $^{-1}$ & $1922(18.7)$ & $0.47(0.35-0.63)$ & $0.32(0.15-0.69)$ & $0.49(0.32-0.75)$ & $0.53(0.33-0.86)$ \\
\hline$>$ Once week $^{-1}$ & 1001 (9.7) & $0.52(0.36-0.76)$ & $0.44(0.18-1.08)$ & $0.63(0.38-1.05)$ & $0.43(0.21-0.88)$ \\
\hline
\end{tabular}

${ }^{*}$ Columns may not total due to missing values. 
Table 3 Distribution of dietary factors at baseline among patients with no previous history of angina ( $n=10522)$, and adjusted relative risks ${ }^{*}(95 \% \mathrm{Cl})$ of death from all causes and from specific causes, associated with frequency of consumption

\begin{tabular}{|c|c|c|c|c|c|c|c|c|c|}
\hline & \multirow[b]{2}{*}{ No. $(\%) \dagger$} & \multicolumn{2}{|c|}{ All-cause mortality } & \multicolumn{2}{|c|}{ IHD mortality } & \multicolumn{2}{|c|}{ Cancer mortality } & \multicolumn{2}{|c|}{ Other mortality } \\
\hline & & Deaths & $\mathrm{RR}(95 \% \mathrm{Cl})$ & Deaths & $\mathrm{RR}(95 \% \mathrm{Cl})$ & Deaths & $\mathrm{RR}(95 \% \mathrm{Cl})$ & Deaths & $\mathrm{RR}(95 \% \mathrm{Cl})$ \\
\hline $\begin{array}{l}\text { Fresh fruit or fruit jui } \\
<1 \text { day week } \\
\text { 1-3 days week } \\
\text { 4-7 days week } \\
\text { 4- }\end{array}$ & $\begin{array}{l}1893(18.5) \\
2726(26.6) \\
5627(54.9)\end{array}$ & $\begin{array}{l}104 \\
142 \\
235\end{array}$ & $\begin{array}{l}1.00 \\
1.07(0.83-1.38) \\
0.84(0.66-1.08)\end{array}$ & $\begin{array}{l}23 \\
31 \\
43\end{array}$ & $\begin{array}{l}1.00 \\
1.18(0.69-2.04) \\
0.84(0.50-1.43)\end{array}$ & $\begin{array}{r}44 \\
60 \\
119\end{array}$ & $\begin{array}{l}1.00 \\
1.01(0.68-1.49) \\
0.91(0.63-1.32)\end{array}$ & $\begin{array}{l}37 \\
51 \\
73\end{array}$ & $\begin{array}{l}1.00 \\
1.10(0.72-1.69) \\
0.75(0.49-1.14)\end{array}$ \\
\hline $\begin{array}{l}\text { Biscuits, cakes, pudd } \\
<1 \text { day week } \\
\text { 1-3 days week }{ }^{-1} \\
4-7 \text { days week }{ }^{-1}\end{array}$ & $\begin{array}{l}2746(27.4) \\
3646(36.3) \\
3645(36.3)\end{array}$ & $\begin{array}{l}158 \\
162 \\
151\end{array}$ & $\begin{array}{l}1.00 \\
0.73(0.59-0.91) \\
0.68(0.54-0.85)\end{array}$ & $\begin{array}{l}35 \\
28 \\
32\end{array}$ & $\begin{array}{l}1.00 \\
0.58(0.35-0.95) \\
0.64(0.39-1.04)\end{array}$ & $\begin{array}{l}67 \\
80 \\
72\end{array}$ & $\begin{array}{l}1.00 \\
0.84(0.61-1.17) \\
0.77(0.55-1.08)\end{array}$ & $\begin{array}{l}56 \\
54 \\
47\end{array}$ & $\begin{array}{l}1.00 \\
0.69(0.48-1.01) \\
0.58(0.39-0.87)\end{array}$ \\
\hline $\begin{array}{l}\text { Fresh or frozen gree } \\
\text { or salad } \\
\quad<3 \text { days week }{ }^{-1} \\
\quad 4-7 \text { days week }^{-1}\end{array}$ & $\begin{array}{l}2569(25.3) \\
7602(74.7)\end{array}$ & $\begin{array}{l}160 \\
318\end{array}$ & $\begin{array}{l}1.00 \\
0.68(0.56-0.83)\end{array}$ & $\begin{array}{l}36 \\
62\end{array}$ & $\begin{array}{l}1.00 \\
0.63(0.42-0.95)\end{array}$ & $\begin{array}{r}67 \\
156\end{array}$ & $\begin{array}{l}1.00 \\
0.77(0.58-1.03)\end{array}$ & $\begin{array}{r}57 \\
100\end{array}$ & $\begin{array}{l}1.00 \\
0.61(0.44-0.85)\end{array}$ \\
\hline $\begin{array}{l}\text { Fresh or frozen fish } \\
\qquad 1 \text { day week } \\
\text { 1-3 days week } \\
\text { 4-7 days week }{ }^{-1}\end{array}$ & $\begin{array}{c}4874(48.6) \\
4663(46.5) \\
495(4.9)\end{array}$ & $\begin{array}{r}203 \\
247 \\
27\end{array}$ & $\begin{array}{l}1.00 \\
1.16(0.96-1.40) \\
1.06(0.71-1.59)\end{array}$ & $\begin{array}{r}36 \\
51 \\
6\end{array}$ & $\begin{array}{l}1.00 \\
1.38(0.90-2.12) \\
1.36(0.57-3.25)\end{array}$ & $\begin{array}{r}103 \\
107 \\
4\end{array}$ & $\begin{array}{l}1.00 \\
0.97(0.74-1.28) \\
1.04(0.59-1.82)\end{array}$ & $\begin{array}{r}64 \\
89 \\
7\end{array}$ & $\begin{array}{l}1.00 \\
1.34(0.97-1.85) \\
0.91(0.42-2.00)\end{array}$ \\
\hline $\begin{array}{l}\text { Fresh or frozen poult } \\
\qquad 1 \text { day week } \\
\text { 1-3 days week } \\
\text { 4-7 days week }{ }^{-1}\end{array}$ & $\begin{array}{c}4191(41.7) \\
5276(52.5) \\
588(5.8)\end{array}$ & $\begin{array}{r}248 \\
203 \\
21\end{array}$ & $\begin{array}{l}1.00 \\
0.83(0.69-1.00) \\
0.76(0.48-1.19)\end{array}$ & $\begin{array}{r}59 \\
32 \\
5\end{array}$ & $\begin{array}{l}1.00 \\
0.63(0.41-0.98) \\
0.95(0.38-2.38)\end{array}$ & $\begin{array}{r}104 \\
107 \\
8\end{array}$ & $\begin{array}{l}1.00 \\
1.00(0.76-1.31) \\
0.63(0.30-1.29)\end{array}$ & $\begin{array}{r}85 \\
64 \\
8\end{array}$ & $\begin{array}{l}1.00 \\
0.74(0.54-1.03) \\
0.85(0.41-1.75)\end{array}$ \\
\hline $\begin{array}{l}\text { Processed meat (e.g } \\
<1 \text { day week } \\
\text { 1-3 days week }{ }^{-1} \\
4-7 \text { days week }^{-1}\end{array}$ & $\begin{array}{l}\text { usages) } \\
6612(66.6) \\
2985(30.1) \\
326(3.3)\end{array}$ & $\begin{array}{r}315 \\
128 \\
15\end{array}$ & $\begin{array}{l}1.00 \\
1.00(0.81-1.23) \\
1.05(0.62-1.76)\end{array}$ & $\begin{array}{r}61 \\
26 \\
4\end{array}$ & $\begin{array}{l}1.00 \\
1.00(0.63-1.60) \\
1.28(0.46-3.54)\end{array}$ & $\begin{array}{r}158 \\
51 \\
8\end{array}$ & $\begin{array}{l}1.00 \\
0.83(0.61-1.15) \\
1.22(0.60-2.51)\end{array}$ & $\begin{array}{r}96 \\
51 \\
3\end{array}$ & $\begin{array}{l}1.00 \\
1.25(0.88-1.77) \\
0.65(0.21-2.07)\end{array}$ \\
\hline $\begin{array}{l}\text { Other fresh or frozen } \\
\text { (e.g. beef, lamb, por } \\
<1 \text { day week } \\
1-3 \text { days week } \\
\text { 4-7 days week } \\
\text { 4-1 }\end{array}$ & $\begin{array}{l}2247(22.1) \\
5393(53.2) \\
2506(24.7)\end{array}$ & $\begin{array}{l}111 \\
242 \\
115\end{array}$ & $\begin{array}{l}1.00 \\
0.86(0.69-1.08) \\
0.71(0.55-0.92)\end{array}$ & $\begin{array}{l}24 \\
48 \\
22\end{array}$ & $\begin{array}{l}1.00 \\
0.78(0.48-1.28) \\
0.55(0.31-0.99)\end{array}$ & $\begin{array}{r}44 \\
121 \\
54\end{array}$ & $\begin{array}{l}1.00 \\
1.11(0.79-1.57) \\
0.88(0.59-1.32)\end{array}$ & $\begin{array}{l}43 \\
73 \\
39\end{array}$ & $\begin{array}{l}1.00 \\
0.66(0.45-0.97) \\
0.63(0.41-0.97)\end{array}$ \\
\hline $\begin{array}{l}\text { Chips } \\
\qquad 1 \text { day week }{ }^{-1} \\
1-3 \text { days week }^{-1} \\
\text { 4-7 days week }{ }^{-1}\end{array}$ & $\begin{array}{c}6475(64.3) \\
3243(32.2) \\
345(3.4)\end{array}$ & $\begin{array}{r}313 \\
136 \\
17\end{array}$ & $\begin{array}{l}1.00 \\
0.99(0.81-1.22) \\
1.17(0.71-1.92)\end{array}$ & $\begin{array}{r}58 \\
27 \\
5\end{array}$ & $\begin{array}{l}1.00 \\
1.08(0.68-1.72) \\
1.69(0.67-4.27)\end{array}$ & $\begin{array}{r}152 \\
64 \\
5\end{array}$ & $\begin{array}{l}1.00 \\
1.00(0.74-1.35) \\
0.78(0.32-1.93)\end{array}$ & $\begin{array}{r}103 \\
45 \\
7\end{array}$ & $\begin{array}{l}1.00 \\
0.94(0.66-1.35) \\
1.33(0.61-2.90)\end{array}$ \\
\hline $\begin{array}{l}\text { Milk } \\
\text { Whole } \\
\text { Semiskimmed } \\
\text { Skimmed }\end{array}$ & $\begin{array}{l}4698(44.9) \\
3380(32.3) \\
2378(22.7)\end{array}$ & $\begin{array}{l}244 \\
151 \\
112\end{array}$ & $\begin{array}{l}1.00 \\
0.93(0.75-1.14) \\
1.01(0.80-1.26)\end{array}$ & $\begin{array}{l}55 \\
33 \\
19\end{array}$ & $\begin{array}{l}1.00 \\
0.94(0.61-1.45) \\
0.85(0.50-1.43)\end{array}$ & $\begin{array}{r}112 \\
71 \\
49\end{array}$ & $\begin{array}{l}1.00 \\
0.92(0.68-1.24) \\
0.90(0.64-1.26)\end{array}$ & $\begin{array}{l}77 \\
47 \\
44\end{array}$ & $\begin{array}{l}1.00 \\
0.93(0.64-1.34) \\
1.27(0.88-1.85)\end{array}$ \\
\hline $\begin{array}{l}\text { Spread } \\
\text { Butter } \\
\text { Hard margarine } \\
\text { Polyunsaturate }\end{array}$ & $\begin{array}{l}3050(29.2) \\
1227(11.7) \\
6172(59.1)\end{array}$ & $\begin{array}{r}185 \\
76 \\
244\end{array}$ & $\begin{array}{l}1.00 \\
1.23(0.94-1.60) \\
0.80(0.66-0.97)\end{array}$ & $\begin{array}{l}32 \\
20 \\
55\end{array}$ & $\begin{array}{l}1.00 \\
2.00(1.14-3.50) \\
1.23(0.79-1.93)\end{array}$ & $\begin{array}{r}87 \\
34 \\
110\end{array}$ & $\begin{array}{l}1.00 \\
1.13(0.76-1.69) \\
0.71(0.53-0.94)\end{array}$ & $\begin{array}{l}66 \\
22 \\
79\end{array}$ & $\begin{array}{l}1.00 \\
1.00(0.61-1.62) \\
0.73(0.52-1.02)\end{array}$ \\
\hline
\end{tabular}


(33.5\%). The distribution of the most frequent causes of death is presented in Table 1.

\section{Baseline reporting}

The baseline distributions of non-dietary exposures and their crude relative risks are presented in Table 2 . Age at entry, current smoking status and the presence of a long-standing illness were all strong predictors of future mortality, whereas regular participation in vigorous exercise was associated with a significant lowering of mortality risk. Subsequent participants in the OXCHECK intervention trial enjoyed significant and substantial reductions in mortality compared with nonparticipants. For each of these exposures, broadly similar point estimates were observed across each of the cause-specific mortality categories. Males and females differed significantly in their smoking at baseline, with proportionally fewer males (27.1\%) than females (48.6\%) reporting they had never smoked, and more males (35.5\%) currently smoking than females $(27.2 \%)(P<0.001)$. While BMI was not associated with overall mortality, participants with a BMI at baseline of 30 and above were at approximately $60 \%$ increased risk of IHD mortality, but were at $40 \%$ lower risk of dying from cancer. Because it was possible that occult cancers may have been contributing to weight loss prior to clinical diagnosis, this analysis was conducted separately during two time periods; the inverse association between BMI of 30 and above and cancer mortality was stronger during the first 5 years of follow-up (RR $0.44,95 \%$ CI $0.19-1.00$ ) than the second 5-year interval (RR 0.69, 95\% CI 0.31-1.50).

\section{Dietary exposures and mortality risk}

Among those with no history of chest pain, the adjusted relative risks of dying associated with dietary exposures are presented in Table 3. For all-cause mortality, small, non-significant elevations in risk were associated with a preference for using hard margarine compared with butter, and frequent consumption of chips. Significant reductions in relative risk were observed for frequent consumption of fresh or frozen green vegetables; biscuits, cakes, puddings or sweets (collectively); and fresh or frozen red meat (beef, lamb, pork). All-cause mortality was substantially reduced among light drinkers, but not among heavier drinkers. Those with regular intakes of fresh fruit or fruit juice, or fresh or frozen poultry had small, non-significant risk reductions for all-cause mortality when compared with infrequent consumers of these items.

Analyses were also conducted relating dietary exposures to specific causes of death (Table 3). Users of hard margarine were at a significant two-fold increased risk of IHD mortality compared with users of butter. Significant reductions in risk of IHD death were observed for those with frequent consumption of 
fresh or frozen green vegetables, and fresh or frozen red meat. Both light drinkers and moderate/heavy drinkers were at substantially reduced risk of IHD death compared with abstainers.

Significantly reduced risks of cancer mortality were observed among those who frequently used polyunsaturated spreads (Table 3). Frequent consumption of fresh or frozen poultry, fresh or frozen green vegetables, polyunsaturated spreads and biscuits, cakes, puddings or sweets were each associated with modest, non-significant risk reductions for cancer mortality.

Reduced risks of death from all other causes were associated with frequent consumption of biscuits, cakes, puddings or sweets, fresh or frozen red meat and fresh or frozen green vegetables. In addition, those

Table 4 Distribution of dietary factors at baseline among patients with no previous history of angina $(n=10522)$ by social class

\begin{tabular}{|c|c|c|c|c|c|}
\hline & No. $(\%)^{*}$ & Classes I-II & Class III & Classes IV-V & Other class $†$ \\
\hline $\begin{array}{l}\text { Fresh fruit or fruit jui } \\
\quad<1 \text { day week } \\
\text { 1-3 days week } \\
\text { 4-7 days week }\end{array}$ & $\begin{array}{l}1893(18.5) \\
2726(26.6) \\
5627(54.9)\end{array}$ & $\begin{array}{r}363(14.4) \\
624(24.8) \\
1534(60.8)\end{array}$ & $\begin{array}{r}841(19.3) \\
1130(25.9) \\
2387(54.8)\end{array}$ & $\begin{array}{l}366(21.4) \\
521(30.5) \\
821(48.1)\end{array}$ & $\begin{array}{l}154(16.1) \\
252(26.4) \\
548(57.4)\end{array}$ \\
\hline $\begin{array}{l}\text { Biscuits, cakes, pud } \\
<1 \text { day week } \\
\text { 1-3 days week } \\
\text { 4-7 days week }{ }^{-1}\end{array}$ & $\begin{array}{l}2746(27.4) \\
3646(36.3) \\
3645(36.3)\end{array}$ & $\begin{array}{l}637(25.7) \\
891(35.9) \\
953(38.4)\end{array}$ & $\begin{array}{l}1121(26.1) \\
1545(36.0) \\
1629(37.9)\end{array}$ & $\begin{array}{l}504(30.4) \\
617(37.3) \\
535(32.3)\end{array}$ & $\begin{array}{l}265(28.5) \\
347(37.4) \\
317(34.1)\end{array}$ \\
\hline $\begin{array}{l}\text { Fresh or frozen gree } \\
\text { or salad } \\
\quad<3 \text { days week }{ }^{-1} \\
\quad 4-7 \text { days week }^{-1}\end{array}$ & $\begin{array}{l}2569(25.3) \\
7602(74.7)\end{array}$ & $\begin{array}{r}496(19.7) \\
2017(80.3)\end{array}$ & $\begin{array}{l}1094(25.1) \\
3258(74.9)\end{array}$ & $\begin{array}{r}531(31.6) \\
1151(68.4)\end{array}$ & $\begin{array}{l}223(23.8) \\
715(76.2)\end{array}$ \\
\hline $\begin{array}{l}\text { Fresh or frozen fish } \\
<1 \text { day week } \\
\quad 1-3 \text { days week }^{-1} \\
\text { 4-7 days week }{ }^{-1}\end{array}$ & $\begin{array}{c}4874(48.6) \\
4663(46.5) \\
495(4.9)\end{array}$ & $\begin{array}{c}1109(44.4) \\
1272(51.0) \\
114(4.6)\end{array}$ & $\begin{array}{c}2102(49.0) \\
2007(46.8) \\
178(4.2)\end{array}$ & $\begin{array}{c}888(53.9) \\
681(41.3) \\
79(4.8)\end{array}$ & $\begin{array}{c}437(47.0) \\
430(46.3) \\
62(6.7)\end{array}$ \\
\hline $\begin{array}{l}\text { Fresh or frozen poul } \\
\qquad 1 \text { day week } \\
\text { 1-3 days week } \\
\text { 4-7 days week } \\
\text { 4-1 }\end{array}$ & $\begin{array}{c}4191(41.7) \\
5276(52.5) \\
588(5.8)\end{array}$ & $\begin{array}{c}849(34.0) \\
1509(60.5) \\
138(5.5)\end{array}$ & $\begin{array}{c}1767(41.0) \\
2318(53.7) \\
228(5.3)\end{array}$ & $\begin{array}{c}852(51.7) \\
699(42.4) \\
98(5.9)\end{array}$ & $\begin{array}{c}423(45.7) \\
430(46.5) \\
72(7.8)\end{array}$ \\
\hline $\begin{array}{l}\text { Processed meat (e.g } \\
\text { sausages) } \\
<1 \text { day week }{ }^{-1} \\
1-3 \text { days week }^{-1} \\
4-7 \text { days week }^{-1}\end{array}$ & $\begin{array}{c}6612(66.6) \\
2985(30.1) \\
326(3.3)\end{array}$ & $\begin{array}{c}1655(66.9) \\
763(30.8) \\
57(2.3)\end{array}$ & $\begin{array}{c}2794(65.5) \\
1340(31.4) \\
134(3.1)\end{array}$ & $\begin{array}{c}1048(64.1) \\
508(31.1) \\
79(4.8)\end{array}$ & $\begin{array}{c}672(75.3) \\
199(22.3) \\
21(2.4)\end{array}$ \\
\hline $\begin{array}{l}\text { Other fresh or frozer } \\
\text { (e.g. beef, lamb, por } \\
<1 \text { day week } \\
\text { 1-3 days week } \\
\text { 4-7 days week }{ }^{-1}\end{array}$ & $\begin{array}{l}2247(22.1) \\
5393(53.2) \\
2506(24.7)\end{array}$ & $\begin{array}{r}516(20.6) \\
1399(55.8) \\
592(23.6)\end{array}$ & $\begin{array}{r}900(20.7) \\
2392(54.9) \\
1064(24.4)\end{array}$ & $\begin{array}{l}414(24.6) \\
867(51.6) \\
399(23.8)\end{array}$ & $\begin{array}{l}236(25.4) \\
420(45.2) \\
274(29.5)\end{array}$ \\
\hline $\begin{array}{l}\text { Chips } \\
\qquad 1 \text { day week }{ }^{-1} \\
1-3 \text { days week }^{-1} \\
\text { 4-7 days week } \text { day }^{-1}\end{array}$ & $\begin{array}{c}6475(64.3) \\
3243(32.2) \\
345(3.4)\end{array}$ & $\begin{array}{c}1595(64.3) \\
814(32.8) \\
73(2.9)\end{array}$ & $\begin{array}{c}2696(62.3) \\
1478(34.1) \\
154(3.6)\end{array}$ & $\begin{array}{c}1077(65.1) \\
515(31.1) \\
62(3.7)\end{array}$ & $\begin{array}{c}673(72.8) \\
226(24.5) \\
25(2.7)\end{array}$ \\
\hline $\begin{array}{l}\text { Milk } \\
\text { Whole } \\
\text { Semiskimmed } \\
\text { Skimmed }\end{array}$ & $\begin{array}{l}4698(44.9) \\
3380(32.3) \\
2378(22.7)\end{array}$ & $\begin{array}{r}1071(42.3) \\
855(33.8) \\
604(23.9)\end{array}$ & $\begin{array}{l}1991(44.8) \\
1424(32.0) \\
1032(23.2)\end{array}$ & $\begin{array}{l}861(49.3) \\
535(30.6) \\
351(20.1)\end{array}$ & $\begin{array}{l}429(43.9) \\
337(34.5) \\
212(21.7)\end{array}$ \\
\hline $\begin{array}{l}\text { Spread } \\
\text { Butter } \\
\text { Hard margarine } \\
\text { Polyunsaturate }\end{array}$ & $\begin{array}{l}3050(29.2) \\
1227(11.7) \\
6172(59.1)\end{array}$ & $\begin{array}{c}716(28.4) \\
185(7.3) \\
1624(64.3)\end{array}$ & $\begin{array}{r}1289(29.0) \\
542(12.2) \\
2608(58.8)\end{array}$ & $\begin{array}{l}502(28.7) \\
281(16.2) \\
965(55.2)\end{array}$ & $\begin{array}{l}303(30.8) \\
107(10.9) \\
573(58.3)\end{array}$ \\
\hline $\begin{array}{l}\text { Alcoholł } \\
\text { Never } \\
\text { Light } \\
\text { Moderate/heavy }\end{array}$ & $\begin{array}{l}1972(19.3) \\
6845(67.1) \\
1391(13.6)\end{array}$ & $\begin{array}{r}282(11.2) \\
1848(73.7) \\
379(15.1)\end{array}$ & $\begin{array}{r}696(16.0) \\
3053(70.0) \\
613(14.1)\end{array}$ & $\begin{array}{l}439(26.1) \\
999(59.5) \\
241(14.4)\end{array}$ & $\begin{array}{c}353(37.2) \\
535(56.4) \\
61(6.4)\end{array}$ \\
\hline $\begin{array}{l}\text { Bread } \\
\text { White bread } \\
\text { Brown bread }\end{array}$ & $\begin{array}{l}4570(44.1) \\
5795(55.9)\end{array}$ & $\begin{array}{r}839(33.5) \\
1667(66.5)\end{array}$ & $\begin{array}{l}1998(45.4) \\
2406(54.6)\end{array}$ & $\begin{array}{l}916(52.7) \\
821(47.3)\end{array}$ & $\begin{array}{l}433(44.5) \\
540(55.5)\end{array}$ \\
\hline
\end{tabular}

${ }^{*}$ Columns may not total due to missing values.

†'Other class' includes those coded as household duties and military servicemen.

†Light alcohol consumption $\leqslant 20$ standard drinks week ${ }^{-1}$ for males; $\leqslant 15$ standard drinks week ${ }^{-1}$ for females. 
who were light consumers of alcohol and those who generally ate brown bread rather than white bread were at significantly lower risks of dying from causes other than IHD or cancer.

\section{Confounding and effect modification}

Because we found a strong association between social class and mortality that may have confounded associations between dietary intake and mortality, we first examined the crude distributions of the main dietary factors by social class (Table 4). Frequent consumption of fresh fruit or fruit juice, fresh or frozen green vegetables, and biscuits, cakes, puddings and sweets were all more common among social classes I-II. In contrast, there was no difference among the social classes for the consumption of either processed meat or fresh or frozen meat. Use of butter was similar among all classes, although use of hard margarine was more prevalent among social classes IV-V.

For four dietary variables which were associated with IHD mortality on preliminary investigation, we then conducted multivariable regression analysis adjusting for age, sex and smoking category, with further inclusion of other potentially confounding factors (including alcohol, social class and all other foods) (Table 5). In general, these adjustments made little substantive difference to the point estimates of relative risk for these dietary exposures (Table 5). Inclusion of terms for social class in models already containing terms for age, sex, smoking and alcohol attenuated the risk estimates associated with consumption of vegetables, and puddings, cakes, biscuits and sweets, but strengthened the association with frequent consumption of fresh or frozen red meat. However, the association of hard margarine and IHD mortality was markedly reduced following adjustment for social class, and was no longer statistically significant.
Several baseline characteristics were identified as strong, independent determinants of mortality in this dataset (see Table 2), the presence of which may have modified the observed effect of the principal dietary determinants of mortality. Within the limits of precision arising from the small group sizes, similar IHD and allcause mortality risk estimates were observed in the presence and absence of each of long-standing disease, participation in the OXCHECK trial or participation in vigorous exercise, for consumption of fresh or frozen green vegetables, biscuits, cakes, puddings or sweets, and fresh or frozen red meat (Table 6).

The possibility that residual confounding by other unmeasured factors could have accounted for these dietary associations with IHD mortality was considered using additional sources of information. Biochemical and physiological data were available for a subset of respondents $(n=2098)$ who returned for a follow-up health check within 1 year of completing this baseline survey. The mean systolic and diastolic blood pressures, and the mean cholesterol, high-density lipoprotein (HDL) and low-density lipoprotein (LDL) levels measured at the 1-year follow-up, classified according to the frequency of consumption of vegetables, puddings, red meat and spreads, are presented in Table 7. For both males and females, there was no substantial variation in either systolic or diastolic blood pressure across the range of food frequencies. Similarly, serum cholesterol levels showed little variation with reported food consumption. Among males, mean HDL and LDL levels increased with reported frequency of eating fresh or frozen red meat, whereas HDL levels declined with increased consumption of biscuits, cakes, puddings or sweets. Among females, there was some evidence for increasing LDL levels with increasing consumption of red meat.

Table 5 Relative risks and $95 \% \mathrm{Cl}$ of IHD mortality, associated with frequent consumption of fresh or frozen green vegetables, puddings, cakes, biscuits or sweets, fresh or frozen red meat and use of hard margarine, after adjustment for various potential confounding factors

\begin{tabular}{|c|c|c|c|c|c|c|c|c|}
\hline \multirow[b]{2}{*}{ Terms included in the model: } & \multicolumn{2}{|c|}{$\begin{array}{l}\text { Fresh or frozen green } \\
\text { vegetables or salad }\end{array}$} & \multicolumn{2}{|c|}{$\begin{array}{l}\text { Puddings, cakes, } \\
\text { biscuits or sweets }\end{array}$} & \multicolumn{2}{|c|}{$\begin{array}{l}\text { Fresh or frozen } \\
\text { red meat }\end{array}$} & \multicolumn{2}{|c|}{$\begin{array}{l}\text { Use of hard } \\
\text { margarine }\end{array}$} \\
\hline & $\mathrm{RR}$ & $95 \% \mathrm{Cl}$ & $\mathrm{RR}$ & $95 \% \mathrm{Cl}$ & $\mathrm{RR}$ & $95 \% \mathrm{Cl}$ & $\mathrm{RR}$ & $95 \% \mathrm{Cl}$ \\
\hline Crude & 0.58 & $0.38-0.87$ & 0.68 & $0.42-1.10$ & 0.82 & $0.46-1.47$ & 1.56 & $0.89-2.72$ \\
\hline Age & 0.54 & $0.36-0.82$ & 0.57 & $0.35-0.92$ & 0.60 & $0.34-1.08$ & 1.98 & $1.13-3.47$ \\
\hline Age, sex & 0.58 & $0.39-0.88$ & 0.53 & $0.33-0.85$ & 0.58 & $0.32-1.03$ & 1.94 & $1.11-3.40$ \\
\hline Age, sex, smoking & 0.63 & $0.42-0.95$ & 0.64 & $0.39-1.04$ & 0.55 & $0.31-0.99$ & 2.00 & $1.14-3.50$ \\
\hline Age, sex, smoking, alcohol & 0.68 & $0.44-1.05$ & 0.63 & $0.38-1.04$ & 0.60 & $0.33-1.08$ & 2.04 & $1.15-3.61$ \\
\hline Age, sex, smoking, alcohol, social class & 0.76 & $0.49-1.20$ & 0.66 & $0.40-1.11$ & 0.57 & $0.30-1.07$ & 1.71 & $0.92-3.16$ \\
\hline Age, sex, smoking, alcohol, fruit & 0.75 & $0.47-1.18$ & 0.57 & $0.34-0.95$ & 0.59 & $0.32-1.08$ & 1.90 & $1.03-3.52$ \\
\hline Age, sex, smoking, alcohol, vegetables & - & - & 0.63 & $0.37-1.05$ & 0.59 & $0.32-1.10$ & 2.12 & $1.15-3.91$ \\
\hline Age, sex, smoking, alcohol, puddings & 0.70 & $0.44-1.18$ & - & - & 0.57 & $0.30-1.08$ & 2.00 & $1.06-3.80$ \\
\hline Age, sex, smoking, alcohol, meat & 0.79 & $0.49-1.26$ & 0.73 & $0.43-1.23$ & - & - & 2.09 & $1.13-3.81$ \\
\hline Age, sex, smoking, other foods ${ }^{*}$ & 0.87 & $0.52-1.46$ & 0.66 & $0.38-1.14$ & 0.60 & $0.31-1.18$ & 1.81 & $0.87-3.79$ \\
\hline
\end{tabular}

"Other foods includes consumption of fresh or frozen green vegetables or salad, fresh or frozen red meat, fresh fruit or fruit juice, puddings, cakes, biscuits and sweets. 
Table 6 Adjusted risk ratios* $(95 \% \mathrm{Cl})$ for mortality from all causes and for IHD, associated with selected dietary screening questions, stratified by independent risk factors

\begin{tabular}{|c|c|c|c|c|c|c|}
\hline & \multicolumn{2}{|c|}{ Participation in OXCHECK } & \multicolumn{2}{|c|}{ Long-standing disease } & \multicolumn{2}{|c|}{ Vigorous exercise } \\
\hline & No & Yes & No & Yes & No & Yes \\
\hline \multicolumn{7}{|l|}{ All-cause mortality } \\
\hline $\begin{array}{l}\text { Vegetables } \\
<3 \text { days week }{ }^{-1} \\
\text { 4-7 days week }{ }^{-1}\end{array}$ & $\begin{array}{l}1.00 \\
0.70(0.49-0.99)\end{array}$ & $\begin{array}{l}1.00 \\
0.71(0.55-0.93)\end{array}$ & $\begin{array}{l}1.00 \\
0.72(0.53-0.97)\end{array}$ & $\begin{array}{l}1.00 \\
0.66(0.51-0.86)\end{array}$ & $\begin{array}{l}1.00 \\
0.70(0.56-0.87)\end{array}$ & $\begin{array}{l}1.00 \\
0.65(0.40-1.07)\end{array}$ \\
\hline $\begin{array}{l}\text { Puddings/biscuits/ca } \\
<1 \text { day week }{ }^{-1} \\
\text { 1-3 days week }{ }^{-1} \\
\text { 4-7 days week } \text { days }^{-1}\end{array}$ & $\begin{array}{l}1.00 \\
0.74(0.50-1.10) \\
0.71(0.47-1.08)\end{array}$ & $\begin{array}{l}1.00 \\
0.71(0.53-0.96) \\
0.64(0.47-0.87)\end{array}$ & $\begin{array}{l}1.00 \\
0.73(0.53-1.02) \\
0.59(0.41-0.84)\end{array}$ & $\begin{array}{l}1.00 \\
0.77(0.57-1.03) \\
0.78(0.58-1.06)\end{array}$ & $\begin{array}{l}1.00 \\
0.75(0.58-0.96) \\
0.66(0.51-0.86)\end{array}$ & $\begin{array}{l}1.00 \\
0.57(0.33-1.00) \\
0.67(0.38-1.16)\end{array}$ \\
\hline $\begin{array}{l}\text { Red meat } \\
<1 \text { day week }{ }^{-1} \\
\text { 1-3 days week } \\
\text { 4-7 days week }{ }^{-1}\end{array}$ & $\begin{array}{l}1.00 \\
1.08(0.73-1.60) \\
0.69(0.43-1.12)\end{array}$ & $\begin{array}{l}1.00 \\
0.93(0.68-1.27) \\
0.69(0.48-1.00)\end{array}$ & $\begin{array}{l}1.00 \\
1.17(0.81-1.69) \\
0.89(0.52-1.25)\end{array}$ & $\begin{array}{l}1.00 \\
0.70(0.53-0.94) \\
0.63(0.45-0.87)\end{array}$ & $\begin{array}{l}1.00 \\
0.92(0.71-1.20) \\
0.71(0.52-0.95)\end{array}$ & $\begin{array}{l}1.00 \\
0.67(0.38-1.17) \\
0.87(0.46-1.64)\end{array}$ \\
\hline $\begin{array}{l}\text { Spread } \\
\text { Butter } \\
\text { Hard margarine } \\
\text { Polyunsaturate }\end{array}$ & $\begin{array}{l}1.00 \\
1.13(0.76-1.68) \\
0.91(0.68-1.21)\end{array}$ & $\begin{array}{l}1.00 \\
1.31(0.91-1.88) \\
0.77(0.59-1.00)\end{array}$ & $\begin{array}{l}1.00 \\
1.10(0.71-1.71) \\
0.92(0.68-1.24)\end{array}$ & $\begin{array}{l}1.00 \\
1.19(0.85-1.69) \\
0.68(0.52-0.88)\end{array}$ & $\begin{array}{l}1.00 \\
1.26(0.93-1.69) \\
0.79(0.63-0.99)\end{array}$ & $\begin{array}{l}1.00 \\
1.23(0.55-2.74) \\
1.19(0.69-2.06)\end{array}$ \\
\hline IHD mortality & & & & & & \\
\hline $\begin{array}{l}\text { Vegetables } \\
<3 \text { days week }{ }^{-1} \\
4-7 \text { days week }^{-1}\end{array}$ & $\begin{array}{l}1.00 \\
0.50(0.25-1.02)\end{array}$ & $\begin{array}{l}1.00 \\
0.74(0.42-1.33)\end{array}$ & $\begin{array}{l}1.00 \\
0.81(0.39-1.68)\end{array}$ & $\begin{array}{l}1.00 \\
0.53(0.31-0.90)\end{array}$ & $\begin{array}{l}1.00 \\
0.68(0.42-1.09)\end{array}$ & $\begin{array}{l}1.00 \\
0.57(0.16-2.01)\end{array}$ \\
\hline $\begin{array}{l}\text { Puddings/biscuits/ca } \\
<1 \text { day week }{ }^{-1} \\
\text { 1-3 days week } \\
\text { 4-7 days week }{ }^{-1}\end{array}$ & $\begin{array}{l}1.00 \\
0.42(0.18-0.99) \\
0.48(0.21-1.12)\end{array}$ & $\begin{array}{l}1.00 \\
0.75(0.38-1.49) \\
0.74(0.37-1.46)\end{array}$ & $\begin{array}{l}1.00 \\
0.58(0.25-1.36) \\
0.62(0.27-1.43)\end{array}$ & $\begin{array}{l}1.00 \\
0.61(0.32-1.17) \\
0.72(0.38-1.34)\end{array}$ & $\begin{array}{l}1.00 \\
0.58(0.33-1.02) \\
0.66(0.39-1.14)\end{array}$ & $\begin{array}{l}1.00 \\
0.47(0.11-1.98) \\
0.49(0.11-2.11)\end{array}$ \\
\hline $\begin{array}{l}\text { Red meat } \\
\quad<1 \text { day week }{ }^{-1} \\
\text { 1-3 days week }{ }^{-1} \\
\text { 4-7 days week }{ }^{-1}\end{array}$ & $\begin{array}{l}1.00 \\
0.96(0.41-2.25) \\
0.67(0.25-1.80)\end{array}$ & Unable to fit model & $\begin{array}{l}1.00 \\
0.93(0.39-2.22) \\
0.50(0.17-1.49)\end{array}$ & $\begin{array}{l}1.00 \\
0.66(0.36-1.22) \\
0.54(0.27-1.08)\end{array}$ & $\begin{array}{l}1.00 \\
0.79(0.46-1.37) \\
0.58(0.30-1.09)\end{array}$ & Unable to fit model \\
\hline Spread & & & & & & \\
\hline $\begin{array}{l}\text { Butter } \\
\text { Hard margarine } \\
\text { Polyunsaturate }\end{array}$ & $\begin{array}{l}1.00 \\
1.84(0.81-4.20) \\
1.33(0.70-2.53)\end{array}$ & $\begin{array}{l}1.00 \\
2.23(1.03-4.82) \\
1.22(0.65-2.28)\end{array}$ & $\begin{array}{l}1.00 \\
1.45(0.51-4.10) \\
1.19(0.56-2.56)\end{array}$ & $\begin{array}{l}1.00 \\
2.33(1.17-4.64) \\
1.21(0.68-2.15)\end{array}$ & $\begin{array}{l}1.00 \\
2.42(1.29-4.53) \\
1.37(0.82-2.29)\end{array}$ & $\begin{array}{l}1.00 \\
1.58(0.26-9.54) \\
1.14(0.29-4.51)\end{array}$ \\
\hline
\end{tabular}

*Stratum-specific risk ratios estimated from Cox proportional hazards model, adjusted for gender, smoking and age group. 
Table 7 Comparison of mean biochemical measurements among 2098 participants who returned for a follow-up health check within 1 year of baseline

\begin{tabular}{|c|c|c|c|c|c|c|c|c|c|c|c|c|}
\hline & \multirow{2}{*}{$\begin{array}{l}\text { Males, } \\
\text { no. (\%) }\end{array}$} & \multirow{2}{*}{$\begin{array}{c}\text { Females, } \\
\text { no. (\%) }\end{array}$} & \multicolumn{2}{|c|}{$\begin{array}{c}\text { Systolic BP } \\
(\mathrm{mmHg})\end{array}$} & \multicolumn{2}{|c|}{$\begin{array}{l}\text { Diastolic BP } \\
\quad(\mathrm{mmHg})\end{array}$} & \multicolumn{2}{|c|}{$\begin{array}{l}\text { Cholesterol } \\
\quad(\text { mean } \\
\left.\text { mmoll }{ }^{-1}\right)\end{array}$} & \multicolumn{2}{|c|}{$\begin{array}{l}\mathrm{HDL} \\
(\text { mean } \\
\left.\text { mmoll }^{-1}\right)\end{array}$} & \multicolumn{2}{|c|}{$\begin{array}{l}\text { LDL } \\
(\text { mean } \\
\left.\text { mmoll }^{-1}\right)\end{array}$} \\
\hline & & & $\mathrm{M}$ & $\mathrm{F}$ & $M$ & $\mathrm{~F}$ & $\mathrm{M}$ & $F$ & $\mathrm{M}$ & $\mathrm{F}$ & $\mathrm{M}$ & $F$ \\
\hline $\begin{array}{l}\text { Fresh or frozen red meat } \\
<1 \text { day week } \\
\text { 1-3 days week } \\
\text { 4-7 days week }{ }^{-1}\end{array}$ & $\begin{array}{l}167(18.4) \\
538(59.1) \\
205(22.5)\end{array}$ & $\begin{array}{l}241(21.9) \\
601(53.3) \\
285(25.3)\end{array}$ & $\begin{array}{l}127.9 \\
130.3 \\
128.1\end{array}$ & $\begin{array}{l}124.0 \\
123.3 \\
124.0\end{array}$ & $\begin{array}{l}76.8 \\
78.3 \\
77.2\end{array}$ & $\begin{array}{l}74.0 \\
74.2 \\
74.0\end{array}$ & $\begin{array}{l}6.1 \\
6.2 \\
6.3\end{array}$ & $\begin{array}{l}6.0 \\
6.1 \\
6.2\end{array}$ & $\begin{array}{l}1.20 \\
1.25 \\
1.29\end{array}$ & $\begin{array}{l}1.55 \\
1.57 \\
1.54\end{array}$ & $\begin{array}{l}3.86 \\
3.94 \\
4.00\end{array}$ & $\begin{array}{l}3.80 \\
3.82 \\
3.89\end{array}$ \\
\hline $\begin{array}{l}\text { Fresh or frozen green vegetables } \\
<3 \text { days week } \\
\text { 4-7 days week } \\
\text {-1 }\end{array}$ & $\begin{array}{l}249(27.3) \\
662(72.7)\end{array}$ & $\begin{array}{l}208(18.4) \\
923(81.6)\end{array}$ & $\begin{array}{l}130.2 \\
129.0\end{array}$ & $\begin{array}{l}121.9 \\
123.9\end{array}$ & $\begin{array}{l}78.0 \\
77.5\end{array}$ & $\begin{array}{l}73.0 \\
74.3\end{array}$ & $\begin{array}{l}6.2 \\
6.2\end{array}$ & $\begin{array}{l}6.1 \\
6.1\end{array}$ & $\begin{array}{l}1.23 \\
1.25\end{array}$ & $\begin{array}{l}1.51 \\
1.57\end{array}$ & $\begin{array}{l}3.96 \\
3.95\end{array}$ & $\begin{array}{l}3.89 \\
3.81\end{array}$ \\
\hline $\begin{array}{l}\text { Biscuits, cakes, puddings, sweets } \\
<1 \text { day week } \\
\text { 1-3 days week } \\
\text { 4-7 days week } \\
\text { 4-1 }\end{array}$ & $\begin{array}{l}212(23.3) \\
319(35.1) \\
379(41.6)\end{array}$ & $\begin{array}{l}299(27.0) \\
421(38.0) \\
388(35.0)\end{array}$ & $\begin{array}{l}131.0 \\
130.6 \\
127.4\end{array}$ & $\begin{array}{l}125.5 \\
120.9 \\
124.4\end{array}$ & $\begin{array}{l}78.1 \\
78.5 \\
76.8\end{array}$ & $\begin{array}{l}75.6 \\
73.0 \\
74.0\end{array}$ & $\begin{array}{l}6.2 \\
6.2 \\
6.1\end{array}$ & $\begin{array}{l}6.1 \\
6.2 \\
6.0\end{array}$ & $\begin{array}{l}1.30 \\
1.23 \\
1.22\end{array}$ & $\begin{array}{l}1.58 \\
1.55 \\
1.56\end{array}$ & $\begin{array}{l}3.87 \\
4.00 \\
3.94\end{array}$ & $\begin{array}{l}3.75 \\
3.88 \\
3.81\end{array}$ \\
\hline $\begin{array}{l}\text { Spreads } \\
\text { Butter } \\
\text { Hard margarine } \\
\text { Soft margarine }\end{array}$ & $\begin{array}{l}264(28.4) \\
116(12.5) \\
551(59.2)\end{array}$ & $\begin{array}{l}307(26.6) \\
106(9.2) \\
743(64.3)\end{array}$ & $\begin{array}{l}129.3 \\
126.5 \\
130.1\end{array}$ & $\begin{array}{l}124.2 \\
121.1 \\
123.7\end{array}$ & $\begin{array}{l}77.0 \\
76.5 \\
78.3\end{array}$ & $\begin{array}{l}74.3 \\
75.1 \\
73.9\end{array}$ & $\begin{array}{l}6.3 \\
6.2 \\
6.1\end{array}$ & $\begin{array}{l}6.2 \\
6.1 \\
6.0\end{array}$ & $\begin{array}{l}1.27 \\
1.28 \\
1.23\end{array}$ & $\begin{array}{l}1.52 \\
1.53 \\
1.57\end{array}$ & $\begin{array}{l}4.06 \\
4.05 \\
3.81\end{array}$ & $\begin{array}{l}3.96 \\
3.89 \\
3.77\end{array}$ \\
\hline
\end{tabular}

$\mathrm{BP}$, blood pressure; cholesterol, non-fasting plasma cholesterol; HDL, high-density lipoprotein cholesterol; LDL, low-density lipoprotein cholesterol.

\section{Discussion}

These prospective data suggest that responses to simple dietary screening questions are associated with risk of subsequent mortality. The principal dietary factors associated with reduced mortality risk in this study were frequent consumption of fresh or frozen green vegetables or salad, fresh or frozen red meat (such as beef, lamb or pork), and biscuits, cakes, puddings or sweets; consumption of these foods was most strongly associated with a reduced risk of IHD mortality. Consumption of hard margarine was associated with a significant increase in risk of IHD mortality, and there was a suggestion that frequent consumption of processed meats (such as pies, burgers and sausages) and chips was associated with modest risk increases, however these latter associations were not statistically significant.

This study was not established to address aetiological hypotheses, and we are not advocating that the diets described by the respondents are necessarily desirable to prevent disease; rather, we have addressed whether a crude tool to assess dietary intake predicts mortality. A priori we had expected that frequent consumption of fresh fruit and fresh or frozen vegetables would be associated with a reduced risk of mortality from IHD and cancer, and that frequent consumption of red meat, biscuits, cakes, puddings and sweets would be associated with increased risks of IHD death. The converse findings for these latter exposures were unexpected, and initially thought to be the result of confounding. Nevertheless, the effects persisted following stratification and adjustment for all other possible confounders for which information was available. For example, the association between total energy intake and survival has been well documented, yet the survival benefits of consuming red meat or puddings and sweets were observed among both active and sedentary participants. While residual confounding cannot be excluded, the magnitude of the association indicates that the effect size of the unmeasured confounding factors would have to be considerable. Either the observed effects for consumption of red meat and puddings and sweets are real, for which we have no clear explanation other than it is a marker for an ill-defined notion of 'well-being' (the 'healthy lifestyle bias' hypothesized by Gillman et al. ${ }^{7}$ ), or else it has arisen by chance. Replication of these analyses using similar survey instruments would clarify this issue.

Despite the volume of the nutritional epidemiological literature, there are few comparable prospective studies $^{8,9}$ which have reported patterns of meat consumption with subsequent risk of myocardial infarction. In the study by Ascherio et al. ${ }^{8}$, the authors reported a non-significant increased risk for those who ate beef four or more times per week compared with those who beef once a month or less (RR 1.38, 95\% CI $0.77-2.29)$. When meat from all sources was pooled, there was little evidence of an increased risk of myocardial infarction (RR 1.18, 95\% CI 0.78-1.80). A prospective study of Seventh Day Adventists ${ }^{9}$ reported an age-adjusted relative risk for non-vegetarians of 1.51 (95\% CI 1.3-1.7) among males, and 1.37 (95\% CI 1.2-1.6) among females. A trend of increasing risk with increasing frequency of meat consumption was 
reported in that study. In a prospective study of British adults, no association was observed between red meat consumption and cancer mortality ${ }^{10}$.

Most investigators have tended to focus on nutritional analyses, the rationale being that this approach provides greater insights into aetiological mechanisms ${ }^{11}$. From the perspective of primary care and public health, the food frequency approach to understanding patterns of morbidity and mortality has practical utility, since the units for analysis (food groups) have direct application to the free-living population.

\section{Possible sources of error}

The response rate to the initial mailing was high, losses to follow-up were very low and outcome information was essentially complete, so it is unlikely that bias arising from selection of an unusual cohort could account for the associations observed. We believe the study population was representative of the practices sampled, although the mortality rate of the cohort overall was slightly lower than that observed for England and Wales owing to 'volunteer bias' (the well-described phenomenon whereby participants in research projects are healthier than non-participants ${ }^{12}$ ).

Misclassification of dietary exposures could have arisen for several reasons. Firstly, the simple dietary instrument used in this survey, including a precategorized food frequency questionnaire, may not truly represent patterns of food consumption in the population. Secondly, the dietary information was collected in a single survey at baseline, and hence will not reflect changes in patterns of dietary intake for the duration of the follow-up. In spite of these potential shortcomings, we have some evidence that this simple dietary instrument has face validity. The same questionnaire was used to survey 9332 Oxfordshire residents in 199192, from which very similar distributions of food consumption were reported ${ }^{13}$. Within the OXCHECK trial, a subset of approximately 2000 participants completed a second dietary assessment (using a similar, but modified, instrument) within 1 year of the baseline survey. The different categorization of food frequencies prevented statistical assessments of repeatability or agreement, nevertheless cross-tabulations of responses to similar dietary questions at baseline and 1 year suggested high levels of reporting consistency (not shown). On the basis of these supportive findings, and since mortality events were prospectively ascertained after the baseline dietary assessment, we have concluded that any misclassification in reporting of dietary exposures is likely to be non-differential with respect to cause of death, and so will tend to reduce the magnitude of any observed association. The decision to exclude those with a previous history of chest pain, who may have recently modified their diets on medical advice, and who were at increased risk of IHD mortality, further reduced the likelihood of systematic reporting errors.

We considered the possibility that dietary intake may have varied according to the presence or absence of other factors, such as energy expenditure and physical exercise, the presence of long-standing illness or medical advice. In particular, since this baseline survey of general practice patients was conducted prior to a health intervention, in which $70 \%$ of our survey respondents participated, and since these participants subsequently enjoyed substantially reduced mortality rates compared with non-participants, there existed a possibility that the observed associations between dietary intake and risk of mortality could be an effect of the health intervention. This was examined in a series of stratified analyses, and the presence or absence of these other factors did not markedly alter the associations. In other words, the association of the simple dietary screening questions with mortality was not simply the result of modification by extraneous factors.

\section{Conclusion}

In conclusion, these data provide some evidence that simple dietary assessments can predict subsequent mortality, particularly from IHD, although it is far from clear whether such associations are causal. While several of the study results were unexpected, most of the survey responses were associated with mortality outcomes in a predictable manner. For example, relative risks for each of the demographic and lifestyle variables (including age, gender, smoking, social class, exercise level, pre-existing disease) and many dietary variables (including consumption of fresh and frozen vegetables, chips, processed meats and alcohol) were in accordance with our prior expectations. In this context, we believe the findings reported here should serve as prompts for review of existing dietary datasets.

\section{Acknowledgements}

Credit for the OXCHECK trial is extended to the former collaborators and steering committee. The authors gratefully acknowledge the contributions of the study participants for their time and efforts. OXCHECK was funded by the Imperial Cancer Research Fund, and David Whiteman is the recipient of a Nuffield Medical Research Fellowship from the University of Oxford.

\section{References}

1 Klor HU, Hauenschild A, Holbach I, Schnell-Kretschmer H, Stroh S. Nutrition and cardiovascular disease. Eur. J. Med. Res. 1997; 2: 243-57.

2 Imperial Cancer Research Fund OXCHECK Study Group. 
Prevalence of risk factors for heart disease in OXCHECK trial: implications for screening in primary care. BMJ 1991; 302: $1057-60$.

3 Rose G, Mccartney P, Reid DD. Self-administration of a questionnaire on chest pain and intermittent claudication. Br. J. Prev. Soc. Med. 1977; 31: 42-8.

4 Coulter A. Lifestyles and social class: implications for primary care. J. R. Coll. Gen. Pract. 1987; 37: 533-6.

5 Office of Population Censuses and Surveys. Classification of Occupations. London: HMSO, 1980.

6 SAS Institute. SAS v6.12. Cary, NC, 1996.

7 Gillman M, Cupples A, Gagnon D, et al. Protective effect of fruits and vegetables. JAMA 1995; 273: 1113-17.

8 Ascherio A, Willett WC, Rimm EB, Giovannucci EL, Stampfer
MJ. Dietary iron intake and risk of coronary disease among men. Circulation 1994; 89: 969-74.

9 Snowdon DA, Phillips RL, Fraser GE. Meat consumption and fatal ischemic heart disease. Prev. Med. 1984; 13: 490-500.

10 Cox BD, Whichelow MJ. Frequent consumption of red meat is not risk factor for cancer. BMJ 1997; 315: 1018.

11 Kromhout D, Bloemberg BPM. Methods in nutritional epidemiology. In: Marmot M, Elliott P, eds. Coronary Heart Disease Epidemiology: from Aetiology to Public Health. Oxford: Oxford University Press, 1992; 140-51.

12 Sackett DL. Bias in analytic research. J. Chron. Dis. 1979; 32: 51-63.

13 Wright L, Harwood D, Coulter A. Health and Lifestyles in the Oxford Region. Oxford: Health Services Research Unit, 1992.

\section{Appendix 1: food frequency questions used in the OXCHECK follow-up study}

About how often do you eat the following foods? (circle one number for each part of the question).

\begin{tabular}{|c|c|c|c|c|}
\hline & Never & $\begin{array}{l}\text { Less than } \\
\text { once a week }\end{array}$ & $\begin{array}{l}1-3 \text { days a } \\
\text { week }\end{array}$ & $\begin{array}{c}\text { 4-7 days a } \\
\text { week }\end{array}$ \\
\hline Fresh fruit or fruit juice & 0 & 1 & 2 & 3 \\
\hline Biscuits, cakes, puddings, sweets & 0 & 1 & 2 & 3 \\
\hline Fresh or frozen green vegetables or salad & 0 & 1 & 2 & 3 \\
\hline Fresh or frozen fish & 0 & 1 & 2 & 3 \\
\hline Fresh or frozen poultry (e.g. chicken) & 0 & 1 & 2 & 3 \\
\hline Processed meat (e.g. pies, burgers, sausages, etc.) & 0 & 1 & 2 & 3 \\
\hline Other fresh or frozen red meat (e.g. beef, lamb, pork) & 0 & 1 & 2 & 3 \\
\hline Chips & 0 & 1 & 2 & 3 \\
\hline Which kind of bread do you eat most of the time? & \multirow{4}{*}{\multicolumn{3}{|c|}{$\begin{array}{l}\text { Wholemeal bread } \\
\text { Other brown bread } \\
\text { White bread } \\
\text { None }\end{array}$}} & 1 \\
\hline & & & & 2 \\
\hline & & & & 3 \\
\hline & & & & 4 \\
\hline Which of the following do you usually spread on your bread? & \multirow{6}{*}{\multicolumn{3}{|c|}{$\begin{array}{l}\text { Butter } \\
\text { Hard margarine (e.g. Stork) } \\
\text { Polyunsaturated margarine (e.g. Flora, sunflower) } \\
\text { Other soft margarine (e.g. Blue Band) } \\
\text { Low fat spread (e.g. Gold, Outline) } \\
\text { None }\end{array}$}} & 1 \\
\hline & & & & 2 \\
\hline & & & & 3 \\
\hline & & & & 4 \\
\hline & & & & 5 \\
\hline & & & & 6 \\
\hline Which type of milk do you use most of the time? & \multirow{5}{*}{\multicolumn{3}{|c|}{$\begin{array}{l}\text { Full cream } \\
\text { Semiskimmed } \\
\text { Skimmed } \\
\text { Coffee whitener (e.g. Coffee-Mate) } \\
\text { None }\end{array}$}} & 1 \\
\hline & & & & 2 \\
\hline & & & & 3 \\
\hline & & & & 4 \\
\hline & & & & 5 \\
\hline
\end{tabular}

